\title{
21
}

\section{Performance Management of Public ATM networks - A Scaleable and Flexible Approach}

\author{
R. Davison, M. Azmoodeh \\ BT Laboratories, \\ Martlesham Heath, Suffolk, UK, \\ rob.davison@bt-sys.bt.co.uk,manooch.azmoodeh@bt-sys.bt.co.uk
}

\begin{abstract}
ATM technology promises a flexible, multi-service network that will support the broadband future. It will need to be robust and efficient in delivering quality of service and this will not be achieved by network protocols alone. Performance management systems will be needed.

The flexibility required from future networks will also be required from their management systems. Approaches derived from the developing technologies of distributed object-oriented computing (CORBA) and distributed artificial intelligence (agents) could allow management systems to be implemented as sets of small-grain, co-operating distributed objects. Such systems will be more flexible, more robust and easier to modify .

This paper presents a framework being developed for understanding performance management functions and an approach to their implementation based on agent technology and the use of CORBA platforms.
\end{abstract}

\section{Keywords}

performance management, ATM, agents, virtual path management

\section{INTRODUCTION}

ATM technology offers the opportunity for constructing networks which can carry many types of service including services beyond those considered when the networks were designed. This will be achieved through the flexibility ATM offers to support diverse bandwidths and quality of services. There are still technological as well as economic uncertainties on how ATM will be developed in the communication networks of the future, however it is already clear that the new flexibilities offered by 
ATM will imply a need for more complex management and control than for conventional single service networks. This is crudely illustrated in figure 1. Each graph represents a space bounded by the number of service types available in a network, the network's efficiency and the quality it offers. Figure 1(a) illustrates how for a conventional network the network design process results in network which can carry few service types with a bounded range of efficiency and quality. The role of network management can be seen as restricting that range further to give the desired quality and efficiency. Figure 1 (b) shows how the possibilities covered by a designed ATM network are much larger and hence network management has to do more to restrict the network to the required behaviours.

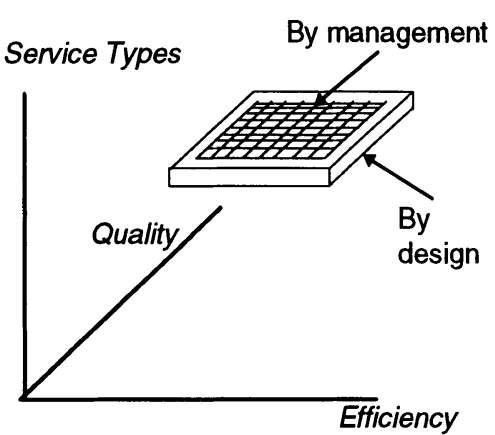

(a) Single Service

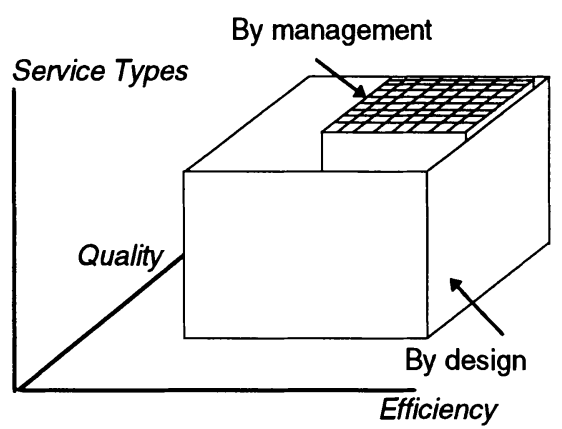

(b) Multi Service Networks

Figure 1 The Behaviour Range of Conventional Networks vs. The Behaviour Range of ATM Networks.

The functional area most greatly affected by the move to multi-service ATM networks is performance management. This is responsible for ensuring that the multiple service types receive their contracted QoS. Sections 2 and 3 of this paper discuss the necessity of performance management functions and the reasons for their complexity. Section 4 describes architectural ideas used to classify and organise the necessary functions to manage the performance of a large ATM network.

The flexibility offered by ATM networks will only be of benefit if it can be matched by flexibility in its management systems. Existing management systems are expensive to maintain and difficult to change, so a major challenge is to devise a management platform which can adapt to the evolving nature of future networks. The fields of distributed object-oriented computing and distributed artificial intelligence could offer benefits here and are discussed in section 5. As part of our ongoing research we have built a management system that manages ATM virtual paths and was designed and implemented using the concepts expounded in this paper. This is discussed in section 6. 
The work described in this paper is based upon a scenario deployment of ATM to support a large, public ATM network carrying a diversity of services including both data and delay-sensitive services. The network will have call control using signalling and its own protocols for traffic and congestion control. Our concern here is the management of the ATM overlay network and not with interactions with lower (e.g. $\mathrm{SDH})$ or higher (e.g. IP) layer management and control functions.

\section{THE NEED FOR ATM PERFORMANCE MANAGEMENT SYSTEMS}

It is often claimed that future networks will be 'self managed'. This term is not properly defined. However, it is usually derived from the 'Internet' style of networks where 'seemingly' there is little management control. Although, strictly speaking this assertion is not true, the intended meaning is that the network protocols (such as IP or ATM protocols) will detect and resolve all performance management issues. We believe that this view ignores two key aspects :

- that control actions are needed at many different time-scales including some that may span hours, days or even weeks and are appropriately addressed by management systems;

- that network providers need the ability to modify a network's behaviour, for instance to increase utilisation, for external business reasons which again requires management functions.

It is certainly true that developing network controls are expanding the range of circumstances that can be coped with by control alone, but there will always be a need for management systems. This does not imply a need for large, centralised systems. The functionality required could be implemented in a highly distributed manner as will be described in section 5 .

A second statement that has been made is that due to development of new technologies, bandwidth as well as switch processor speed and buffer capacities will be so plentiful that performance management is unnecessary. It is fairly obvious that for lightly loaded networks, congestion is less likely to occur, so a suggestion would be that as loading increases the resources (bandwidth, buffer capacities, processor's speed) available should be increased to avoid congestion problems. As expanded in (Jain, 1992) and (Jain, 1990), this is likely to move the point of congestion (for instance from an overloaded circuit to an overloaded buffer). It may be argued that a balanced re-configuration - where all nodes and links are upgraded -would avoid this problem. However, the burstiness of traffic sources will mean that simultaneous bursts can occur, resulting in worst case scenarios that are dramatically different from normal conditions. A network designed to support these extreme cases is unlikely to be economically viable, so some form of management is necessary to cope with these cases. 
An interesting area of study is the trade-off between the cost of implementing management systems and the costs of network hardware. Currently, network utilisation is a key factor for providers. However it is possible that as network hardware costs fall, it will be cheaper to accept lower network utilisation in return for less active performance management systems.

\section{SOURCES OF COMPLEXITY IN ATM PERFORMANCE MANAGEMENT}

Having justified the need for ATM performance management systems we move on to address why ATM performance management poses new challenges. Several fundamental aspects of ATM have a significant impact on the functionality required from network management systems. The most important are:

\section{Shared physical resources and virtual reservation}

In the PSTN, when a call is set up, physical resources are reserved to support that call during its lifetime. In contrast, in ATM networks virtual resources (VPCs) are reserved and the underlying physical resources are shared. An important consequence is that rather than the result of congestion being call barring as it would be in today's PSTN, the result of congestion will be downgrading of the quality of existing connections. This is a very different behaviour that will impact upon network management.

\section{Diverse connection types in a single network}

A number of different connection types are being defined for ATM networks each able to support different kinds of services. Examples are Constant Bit Rate connections that require the same bandwidth throughout their life and Available Bit Rate connections that use flow control techniques to match the bandwidth they can use to the bandwidth available in the network. The different connection types all share the same cell structure and can be carried by the same network. However, their behaviours are fundamentally different in terms of how they use resources, the traffic contracts they will have and how they act in the event of congestion. So a single ATM network will contain connections that behave in very different ways, will impact upon each other and yet will need to be managed as part of a single system.

\section{Managing switch buffers}

The asynchronous nature of ATM is enabled by placing buffers in switches that cope with short term variations in load. The size of the buffers and the strategies used to partition and empty them will impact upon quality of service and upon the efficiency that can be produced from the network. Although it is unclear how dynamically these will need to be changed, it seems likely that some management intervention will be needed. 


\section{Complex traffic patterns make prediction difficult}

An important benefit of ATM is that it provides the ability for users to send highly variable bit-rates into the network providing they stay within agreed parameters such as, for example, mean and peak transmission rates. This does mean that functionality that depends on being to able to predict network usage, such as accepting new connections or preventing congestion by re-routing, are complicated since such predictions will be statistical with potentially large inaccuracies.

Standards bodies such as ATM-F, ITU and ETSI have, so far, dealt primarily with near term issues in the deployment of ATM, such as connection management and fault management. The challenging area of performance management has yet to be adequately addressed by these groups. (ATM-Forum)(ITU-T, I.371)

\section{A FRAMEWORK FOR DESIGNING ATM PERFORMANCE MANAGEMENT SYSTEMS}

This section describes architectural ideas we have been using to understand, specify and start to implement ATM performance management systems.

\subsection{Two aspects of performance management functions}

The actions taken by performance management systems can be divided into two categories: those actions taken in response to impending network congestion or QoS problems and those taken to modify the behaviour of the network system as a result of some business decision. Figure 2 represents this division by representing the two categories as 'direction setting' and 'direction enforcing'.

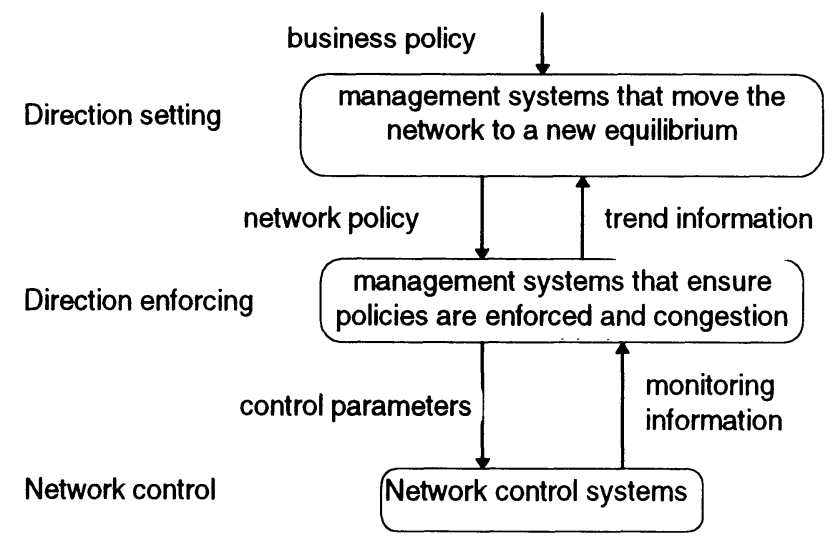

Figure 2 The two aspects of performance management functionality. 
Direction setting functionality would be responsible for converting a business policy such as providing a new connection type or increasing network utilisation - into its impacts upon different parts in the network. These impacts are then passed on as network policy statements to the direction enforcing functions which use them to configure and manage network controls and resources. Our work to date has concentrated on direction enforcing management functions which are the topic of the rest of this paper.

\subsection{Different layers of traffic variation}

It has been widely recognised that cell, burst, and call level traffic variations need to be studied and controlled separately (Key)(Hui). (Arvidsson) takes this approach further and defines further traffic variations more of interest to management functions. We believe the use of time-scales to aid understanding is useful and have derived our own time-scales as defined in table 1.

Table 1 Time-scale of events occurring in an ATM network

\begin{tabular}{|c|c|c|}
\hline Layer & Variation & Example \\
\hline $\begin{array}{l}\text { Permanent } \\
\text { Environmental } \\
\text { /Network }\end{array}$ & $\begin{array}{l}\text { Changes due to permanent change } \\
\text { in user population/sub-population } \\
\text { behaviour and changes due to } \\
\text { permanent change in the network }\end{array}$ & $\begin{array}{l}\text { reduced tariffs, } \\
\text { new service types }\end{array}$ \\
\hline Temporary & Changes due to temporary change in & phone-in, periodic \\
\hline $\begin{array}{l}\text { Environmental } \\
\text { /Network }\end{array}$ & $\begin{array}{l}\text { user population/sub-population } \\
\text { behaviour and changes due to } \\
\text { temporary change in the network }\end{array}$ & $\begin{array}{l}\text { change such as } \\
\text { weekday } \mathbf{v} \\
\text { weekend }\end{array}$ \\
\hline Connection & Change due to use of a connection & $\begin{array}{l}\text { shutting it down, } \\
\text { changing its QoS }\end{array}$ \\
\hline Activity & $\begin{array}{l}\text { Changes due to an application level } \\
\text { change in the use of a connection }\end{array}$ & $\begin{array}{l}\text { change of scene in } \\
\text { a video or } \\
\text { downloading next } \\
\text { page in a } \\
\text { document reading } \\
\text { application }\end{array}$ \\
\hline Burst & $\begin{array}{l}\text { changes due to variations in bursts } \\
\text { at either the user terminal or inside } \\
\text { network due to a particular } \\
\text { combination of bursts }\end{array}$ & $\begin{array}{l}\text { packetisation } \\
\text { effects, higher } \\
\text { level flow control }\end{array}$ \\
\hline Cell & changes due to cell level variations & $\begin{array}{l}\text { segmentation into } \\
\text { cells, shaping and } \\
\text { policing functions }\end{array}$ \\
\hline
\end{tabular}

The time-scales were used to capture the different causes of traffic variation in an ATM network. These causes could then be grouped together to form the problems that 
a particular network management function should solve. This use of time-scales does not necessarily imply that those functions aimed at solving problems at a particular time-scale have to operate within that time-scale. For instance, a source policing algorithm would have to work at speeds to match cell rates however a management function that set parameters for such an algorithm would work on a much longer timescale considering trends in traffic measurements. This example illustrates why the time-scales are used to organise network problems rather than being applied to structuring network management functions and is a key difference from (Arvidsson)

\subsection{Analysis vs. tuning}

A further structuring concept that we have found useful is to break some identified functions into an analytical part and a tuning part. The analytical part takes a more global view and uses analytical techniques to produce a new solution, whereas the tuning part takes a more local, dynamic view and tries to tune parts of a solution. The algorithms used in the analytical part can take in data from a large part or the whole of the network and can have time and space to process this data to produce near-optimal solutions. However we believe that there is a need for a more dynamic form of management that is able to respond more quickly if less optimally. The tuning part only monitors and changes a small part of the network so is able to respond and to maintain a satisfactory state. Eventually, in all cases, variations will be sufficiently great and will require analysis.

The example in section 6 illustrates this approach.

\section{TECHNOLOGY TO RE-ARCHITECT NETWORK MANAGEMENT}

The flexibility offered by ATM networks will only be of benefit if it can be matched by flexibility and adaptability in its management systems. Existing management systems are expensive to maintain and difficult to change, so a major challenge is to devise a management platform which can adapt to the evolving nature of future networks. As part of our work we have been considering two technologies to aid in this goal - objectoriented distributed computing platforms and distributed artificial intelligence.

\subsection{CORBA-based network management}

The TMN architecture (ITU-T, M3010) has provided an implementation architecture offering benefits due to structuring and distributing functions. However, since its conception, distributed computing technology has developed significantly enabling :

- a finer grained distribution with benefits of re-use, maintainability and scalability;

- a higher level API, rather than a protocol interface, for application programmers to use.

The developing CORBA standard is an attempt to provide a standardised approach to distributed computing and is seen by many as a key technology in the future of 
communication management. (OMG)(TINA-C). Designers of network management systems can progress from an implementation where the unit of distribution is a large system offering many managed objects at its interfaces to one where the unit of distribution is a small object which offers a few interfaces to manage perhaps as little as a single resource. Figure 3 illustrates this possibility and is derived from (ITU-T, X.703) and (OMG). It shows how a single, large Operations Systems Function in a TMN could be replaced by many, smaller systems in a CORBA-based solution.
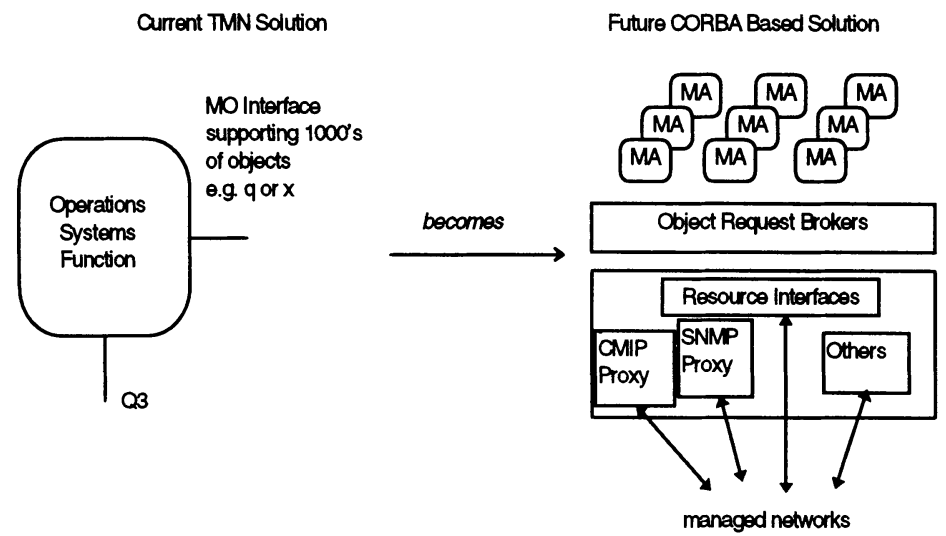

Figure 3 Conventional Management Systems vs. a CORBA based Management System

This picture is a possible future rather than an implementable present. Concerns still exist over CORBA technology in areas including performance, scalability and security, but we believe this technology represents a key part of the future.

\subsection{Distributed artificial intelligence}

The field of Distributed Artificial Intelligence (DAI) and particularly agent technology has suffered from producing more hype than content, however it does contain genuinely useful ideas which could benefit the network management domain. The previous section describes how management systems could be built of small management applications, each encapsulated as an object on a distributed CORBA platform. DAI ideas and techniques could extend this idea to move from a hierarchically structured architecture such as TMN to one based upon peer-to-peer interaction. This would give a more loosely coupled system that could be more flexible and adaptable.

This technology is at an early stage but early applications to complex domains such as air-traffic control (Ljungberg) and telecommunications (Azarmi) suggest fruitful possibilities 


\section{APPLICATION TO VIRTUAL PATH BANDWIDTH MANAGEMENT}

As part of our work we are implementing and experimenting with demonstrators which are being designed and constructed using the ideas described above. At the time of writing, the best example is a system produced to perform virtual path bandwidth management. Its overall aim is to ensure that the set of virtual paths set up in a network are able to deliver both the appropriate quality of service to individual connections whilst maintaining the connection acceptance rates desired by the provider. This is a non-trivial task because it depends upon the call patterns of users and their traffic patterns during calls, both of which are complex and difficult to estimate. The system acts in the Temporary Environmental/Network time-scale defined in section 4.2 dealing with variations over a number of users and hence traffic variations over hours or days. Examples may be different call patterns at different times of the day or week.

A conventional approach to the problem might be to design and implement a single algorithm that takes in the estimate for traffic volumes and produces a complete VP network. Such a system would go against our aims of highly distributed and flexible systems. Instead we use the ideas of analysis vs. tuning described in section 4.3 to produce a system that consists of a design algorithm that is a long-term function with a global view and a set of tuning functions that work quickly and flexibly with only local views. Although both are looking at problems caused in the 'temporary environmental/network' time-scale, the tuning functions operate on the network on a frequent basis whilst the design algorithm operates on a weekly or monthly basis.

The VP bandwidth management system comprising the VP network design function and tuning agents is illustrated in figure 4.

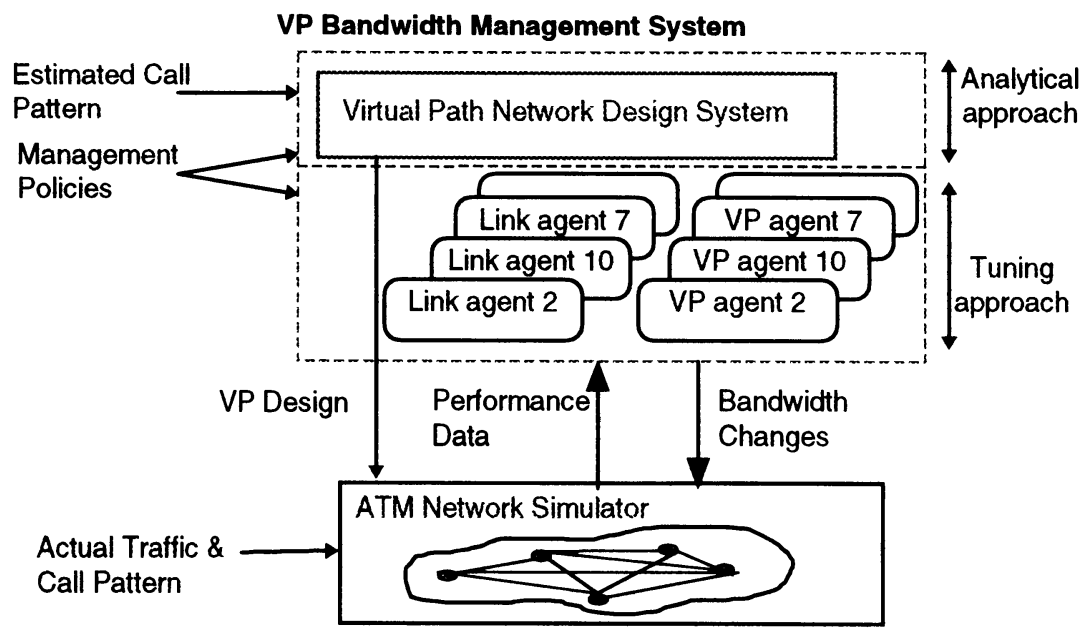

Figure 4 The organisation of the VP bandwidth management system 
The ideas described in section 5 were used to implement the tuning functions as a set of agents each responsible for a different resource in the network and only having dynamic knowledge or control of that resource. By communicating with each other they are able to modify VP bandwidths in the network avoiding potential congestion. It is important to notice that, in an agent-like manner, there is no type of agent that does VP bandwidth management, rather the behaviour of VP bandwidth management emerges from their distributed interaction.

The agents were constructed to control a public ATM network simulator produced by the RACE ICM project (RACE ICM). This simulator provides near-real-time simulation and provides a management interface allowing network data to be extracted during simulation and limited management controls to be applied. Experiments so far suggest that the agents described do achieve the goal of increasing call acceptance although they have yet to be properly bench-marked. The graph in figure 5 illustrates this and is based on a simulated network of 4 ATM nodes, interconnected by 5155 Mbps transmission links supporting 11 VPCs. Three connection types were supported: $1 \mathrm{Mbps}$ CBR, VBR with mean of $3 \mathrm{Mbps}$ and peak of $5 \mathrm{Mbs}$ and VBR with mean of 4 Mbps and peak of $6 \mathrm{Mbps}$. Traffic volume in the network consists of order of 50 users for each source-destination pair, generating calls based on call pattern with mean holding time of $110 \mathrm{sec}$ and mean silence time of $130 \mathrm{sec}$. For each connection, traffic is generated as a set of bursts with rates based on truncated negative exponential distribution.

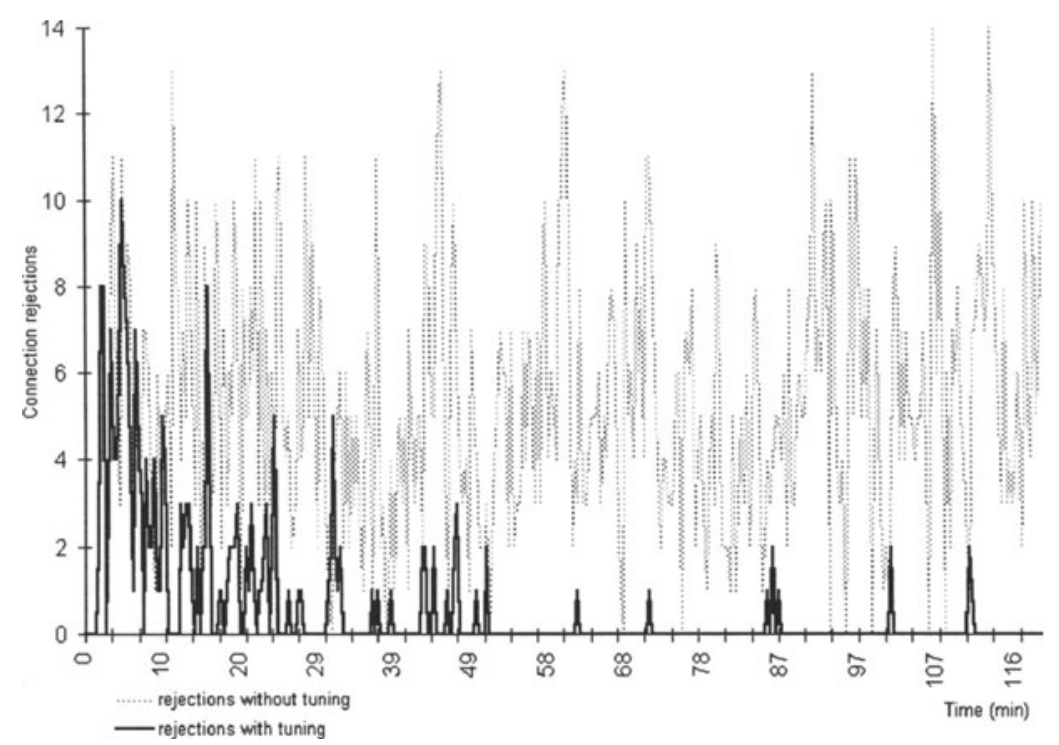

Figure 5 Connection Rejection with and without tuning agents 
Each agent contains a very simple rule-based system that provides its behaviour. An expected benefit of this approach was that the required behaviour would be provided by many, small systems which should be easier to maintain and modify. However in practice we found that although the 'micro' aspect of the agents was simpler, the 'macro' aspects were much more difficult owing to unfavourable interactions such as deadlock, infinite loops, and inappropriate actions. This highlighted the need for development tools that cope with distribution. A second factor that needs further research concerns the granularity of agents. The system that we built does have very small agents which may result in higher levels of messaging overhead between the agents and a greater configuration problem. There is a design trade-off to be understood which balances the benefits of smaller agents against the resulting overheads.

\section{CONCLUSIONS}

ATM performance management is a vital area that needs to be addressed if ATM is to provide a flexible, multi-service network platform. Network controls alone will not be sufficient.

A time-scale based framework has been presented that categorises network problems and events according to the traffic variation they cause. This framework is being used to capture the functions that are needed to manage ATM networks and has been found to be useful.

Communication management systems are already becoming the major barrier to change, preventing rapid deployment of new services and functions. For this situation to be changed, new approaches are needed that will result in smaller and more flexible systems that are cheaper to build, change and discard. Initial work on using the technologies of DAI and CORBA offers promise in achieving this goal.

The ideas described in this paper are being developed and enhanced as part of an ongoing programme of work aimed at producing proven specifications and designs for a new breed of ATM performance management systems.

\section{Acknowledgements}

The authors would like to thank Sagar Gordhan of BT for his support in producing software to monitor the ATM simulator and for his experimental analysis of the VP bandwidth management demonstrator. 


\section{REFERENCES}

Arvidsson A. (1995) "On the usage of virtual paths, virtual channels, and buffers in ATM traffic management", $12^{\text {th }}$ Nordic Teletraffic Seminar NTS12, Finland 22-24 Aug 1995.

ATM-Forum Traffic Management Specification V4.0, ATM-F/95-0013R11

Azarmi N., Nwana H, Smith R (1996) Special Edition on Intelligent Software Systems, BT Technology Journal, vol. 14 no. 4 October 1996

Hui J Y (1988) "Resource allocation for broadband networks", IEEE journal of selected areas in communication, 6, 1988.

ITU Draft Recommendation X.703, Open Distributed Management Architecture

ITU-T Recommendation I.371, "Traffic Control and Congestion Control in B-ISDN", Perth, November 1995

ITU-T Recommendation M3010 Principle for a Telecommunications Management Network

Jain R. (1990) "Congestion control in computer networks : issues and trends" IEEE Network Magazine, May 1990, pp24-30

Jain R. (1992) "Myths about congestion management in high speed networks", Internetworking: Research and Experience, Vol 3, pp 101-113.

Key P. (1995) "Connection Admission Control in ATM networks" BT Technology Journal, 13, 3, July 1995.

Ljungberg and Lucas (1992) "The Oasis air-traffic management system", Proceedings of the Second Pacific Rim Conference on AI, 1992

OMG Telecom Special Interest Group (1996) "CORBA-based Telecommunication Network Management” White Paper, Draft 3.0, Feb 1996

RACE Project R2059 ICM, http://gryphon.elec.qmw.ac.uk/ icm

TINA-C (1994), Overall Concepts and Principles of TINA, TINA Del. TB_MDC.018_1.0_94

Rob Davison graduated from Bristol University, UK, in Computer Systems Engineering and joined BT Labs in 1988. He has worked in the field of communications management since 1990 with a particular emphasis on the application of AI techniques. He currently leads a team of researchers addressing network management with focus on novel architectures for network management, ATM performance management and the application of $\mathrm{AI}$.

Manooch Azmoodeh graduated from the University of Manchester, UK, with a Ph.D in computer science. He spent five years at the University of Essex researching intelligent databases and query languages and then joined BT Labs in 1988. He has researched various aspects of communication management systems, with emphasis on the application of $\mathrm{AI}$ and database techniques. His current interests are new network management architectures based on emerging software technologies and their application to broadband networks. 